\title{
Improvement of biocomposite properties based tapioca starch and sugarcane bagasse cellulose nanofibers
}

\begin{abstract}
Biocomposite based tapioca starch (TS) and sugarcane bagasse cellulose nanofibers (SBCN) was made through casting method. SBCN was prepared by chemical and ultrasonication process. It was successfully displayed by transmission electron microscope (TEM) in range 20 - $45 \mathrm{~nm}$. Meanwhile, particle size analysis (PSA) also supported the distribution diameter of SBCN for $59.75 \pm 10.84 \mathrm{~nm}$. SBCN and glycerol were used as reinforcement and plasticizer, respectively. The amount concentration of SBCN was varied from 0 to $8 \mathrm{wt} \%$. Biocomposite was characterized by using scanning electron microscopy (SEM) and tensile test. SEM image displays SBCN is in good interfacial bonding with the matrix. The highest tensile strength of biocomposite was in TS/4SBCN sample for $20.84 \mathrm{MPa}$. These results showed that SBCN fiber become potential candidate as reinforcement in biocomposite application.
\end{abstract}

Keyword: Biocomposite; Cellulose nanofibers; Sugarcane bagasse fiber; Tapioca starch; Tensile 